\title{
Apelin-36 protects against lipopolysaccharide-induced acute lung injury by inhibiting the ASK1/MAPK signaling pathway
}

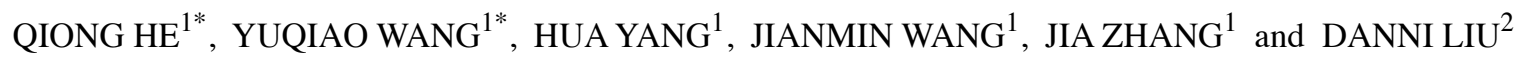 \\ Departments of ${ }^{1}$ Critical Care Medicine and ${ }^{2}$ Cardiology, People's Hospital of Ningxia Hui Autonomous Region \\ (Ningxia Medical University Affiliated Autonomous Region People's Hospital), Yinchuan, Ningxia 750002, P.R. China
}

Received June 15, 2020; Accepted September 30, 2020

DOI: $10.3892 / \mathrm{mmr} .2020 .11644$

\begin{abstract}
Apelin-36 is able to mediate a range of effects on various diseases, and is upregulated in lipopolysaccharide (LPS)-induced acute lung injury (ALI). However, to the best of our knowledge, whether apelin-36 is able to regulate LPS-induced ALI has yet to be investigated. The present study aimed to investigate the role of apelin-36 in LPS-induced ALI, and the putative underlying mechanisms. Rats were assigned to one of four treatment groups: The Control group, apelin-36 group, LPS group and LPS + apelin-36 group. At $4 \mathrm{~h}$ after intratracheal instillation of LPS $(5 \mathrm{mg} / \mathrm{kg})$, rats were intraperitoneally treated with $10 \mathrm{nmol} / \mathrm{kg}$ apelin-36. Subsequently, pathological manifestations and the extent of inflammation and apoptosis of the lung tissues were assessed. Untransfected and apoptosis signal-regulating kinase 1 (ASK1)-overexpressing Beas-2B cells were treated with LPS in the absence or presence of apelin-36, and subsequently the levels of inflammation and apoptosis were assessed. The results obtained showed that the level of apelin-36 was increased in the bronchoalveolar lavage fluid (BALF) of LPS-treated rats. Co-treatment with apelin-36 alleviated LPS-induced lung injury and pulmonary edema, reduced the levels of pro-inflammatory cytokines, including interleukin-6, monocyte chemoattractant protein-1 and tumor necrosis factor- $\alpha$, in BALF, and inhibited apoptosis in the lung tissues. The presence of apelin-36 also blocked the activation of LPS-induced ASK1, p38, c-Jun $\mathrm{N}$-terminal kinase and extracellular signal-regulated kinase in lung tissues. In vitro studies performed with Beas-2B cells showed that the addition of apelin-36 led to an increase
\end{abstract}

Correspondence to: Dr Danni Liu, Department of Cardiology, People's Hospital of Ningxia Hui Autonomous Region (Ningxia Medical University Affiliated Autonomous Region People's Hospital), 301 Zhengyuan North Street, Jinfeng, Yinchuan, Ningxia 750002, P.R. China

E-mail: liudanni112@163.com

*Contributed equally

Key words: acute lung injury, apelin-36, apoptosis, lipopolysaccharide, mitogen-activated protein kinase, inflammation in the cell viability of LPS-induced Beas-2B cells in a concentration-dependent manner. Additionally, co-treatment with $1 \mu \mathrm{M}$ apelin-36 prevented LPS-induced inflammation and apoptosis. However, overexpression of ASK1 significantly reversed the inhibitory effects of apelin-36 on LPS-induced inflammation and apoptosis. Taken together, the results of the present study demonstrated that apelin-36 was able to protect against LPS-induced lung injury both in vivo and in vitro, and these actions may be dependent on inhibition of the ASK1/mitogen-activated protein kinase signaling pathway.

\section{Introduction}

Acute lung injury (ALI) and acute respiratory distress syndrome (ARDS) are major clinical syndromes of acute respiratory failure that can be caused by infectious factors, such as bacterial and viral infections, and non-infectious factors, such as inhalation of toxic gas, blood transfusion, drug poisoning and acute pancreatitis $(1,2)$. ALI may lead to alveolar epithelium or vascular endothelial cell damage, and an increase in pulmonary vascular permeability (1). However, the pathogenesis of ALI is complicated, and among the various putative mechanistic explanations, an increased production of pro-inflammatory factors in the lungs is currently recognized as the most important mechanism (2). The increased release and activation of inflammatory cytokines can lead to an uncontrolled inflammatory response, causing damage to the pulmonary capillary endothelial cells and alveolar epithelial cells (2). Damaged endothelial cells release reactive oxygen species, which subsequently leads to an increase in the permeability of lung endothelial cells, allowing large quantities of protein-rich substances to enter the bronchial tubes and alveolar cavities, causing pulmonary edema, thereby reducing the effectiveness of lung ventilation, and even leading to respiratory failure $(3,4)$. There are currently no specific drugs available for the treatment of ALI. Therefore, the development of effective treatment strategies is of great clinical significance in treating ALI.

The apelin family comprises a class of endogenous active peptides with different molecular structures encoded by the same gene. These peptides function as endogenous ligands for the seven-transmembrane G-protein-coupled receptor, APJ (5). The precursor of the apelin peptides is a polypeptide composed of 77 amino acids encoded by the $\mathrm{X}$ chromosome, 
which is hydrolyzed into active polypeptides of different lengths by proteases (6). These active polypeptides can be classified according to their number of amino acids; peptides produced are typically 36,17 and 13 amino acids in length, in addition to other subtypes. The longer the peptide chain, the stronger the binding of the apelin peptide to the APJ receptor (5). One such naturally occurring apelin peptide comprising 36 amino acid residues is termed 'apelin-36'. Apelin-36 is a long peptide fragment of the apelin precursor protein that is widely distributed in the central nervous system and in peripheral tissues (7). Studies have demonstrated that apelin-36 is closely associated with a variety of diseases, including nervous system diseases, myocardial ischemia and diabetes (8-12). A study by Fan et al (5) revealed that the levels of apelin-13 and apelin-36 were increased in the plasma, lung tissues and bronchoalveolar lavage fluid (BLAF) following lipopolysaccharide (LPS)-induced lung injury, and the addition of apelin-13 could reduce LPS-induced lung injury. However, further studies are required to further elucidate the underlying mechanisms. Furthermore, a recent study demonstrated that apelin-36 could regulate oxidative stress, autophagy and apoptosis to exert neuroprotective effects via inhibiting the apoptosis signal-regulating kinase 1 (ASK1)/c-Jun N-terminal kinase (JNK)/caspase-3 apoptotic pathway in a mouse model of Parkinson's disease (13). In the present study, the aim was to investigate whether apelin-36 could regulate LPS-induced ALI both in vivo and in vitro, as well as exploring the potential mechanisms of apelin-36 in an LPS-induced ALI rat model.

\section{Materials and methods}

Animals and regents. A total of 25 male Sprague-Dawley rats (age, 7-8 weeks) weighing 250-300 $\mathrm{g}$ were purchased from the Nanjing Jiancheng Bioengineering Institute. All animals were kept in a specific pathogen-free environment at $25^{\circ} \mathrm{C}$ under a controlled 12/12 h light/dark cycle, and all rats received food and water ad libitum. All experimental procedures were carried out in accordance with the US National Institutes of Health Guidelines for the Care and Use of Laboratory Animals, and were approved by the Ethical Committee on Animal Research at People's Hospital of Ningxia Hui Autonomous Region (approval no. IACUC-20191002-10; Yinchuan, China).

LPS was also obtained from Nanjing Jiancheng Bioengineering Institute. Apelin-36 was purchased from Phoenix Pharmaceuticals, Inc. The apelin-36 kit (cat. no. kt98790) was provided by MSK Biotechnology Co., Ltd., whereas the ELISA kits and antibodies were obtained from Abcam.

LPS-induced ALI. Rats were randomly assigned to four treatment groups, with 5 rats in each group, as follows: i) Control group; ii) apelin-36 group; iii) LPS group; and iv) LPS + apelin-36 group. Apelin-36 was intraperitoneally injected into rats at a concentration of $10 \mathrm{nmol} / \mathrm{kg}$ for $24 \mathrm{~h}$, in accordance with a previous study (5). For induction of ALI, rats were intratracheally instilled with $5 \mathrm{mg} / \mathrm{kg}$ LPS for $24 \mathrm{~h}$, whereas the control animals were instilled intratracheally with an equal volume of normal saline, as previously described $(14,15)$. For the LPS + apelin-36 co-treatment group, apelin-36 was administered $4 \mathrm{~h}$ after LPS instillation. At $24 \mathrm{~h}$ after the LPS or apelin-36 treatment, rats were anesthetized using $1 \%$ pentobarbital $(50 \mathrm{mg} / \mathrm{kg}$, intraperitoneal injection) for the subsequent experiments.

Collection of BALF. After treatment with LPS for $24 \mathrm{~h}$, the rats were anesthetized using $1 \%$ pentobarbital $(50 \mathrm{mg} / \mathrm{kg}$, intraperitoneally injection) and sacrificed by cervical dislocation. After exposing the chest cavity and intubating the trachea, PBS was slowly dropped into the lung bronchus, and the BALF was collected for further analysis, as described previously (16).

Detection of the lung wet/dry ratio. Following sacrifice, the lungs of the rats were isolated, and the wet lungs were harvested and subsequently weighed to obtain the wet weight. The blood on the lungs was then blotted dry, and the lungs were dried in an $80^{\circ} \mathrm{C}$ incubator for $48 \mathrm{~h}$, after which the weight of the lungs was recorded as the dry weight. The lung wet/dry weight ratio was then calculated (wet weight/dry weight), and used to assess the degree of pulmonary edema.

Histological staining. To evaluate the histological alterations, lung tissues were fixed with $4 \%$ paraformaldehyde at $4^{\circ} \mathrm{C}$ for $24 \mathrm{~h}$, embedded in paraffin, and $4-\mu \mathrm{m}$ thick sections were cut. After deparaffinization and dehydration, the sections were stained with hematoxylin and eosin (Beyotime Institute of Biotechnology) at room temperature for 2 min using standard histological techniques, and were subsequently observed under an optical microscope for pathological examination. Furthermore, some of the tissue sections were deparaffinized, rehydrated and subjected to TUNEL (Beyotime Institute of Biotechnology) staining, three fields of view were examined and the extent of cellular death was determined using a fluorescence microscope (Olympus Corporation). DAPI was used to stain the nuclei (blue) at room temperature for $1 \mathrm{~min}$. The TUNEL positive cells were calculated using the following formula: TUNEL positive cells=the number of green cells/total (blue) cells $\times 100 \%$, this was determined using ImageJ 1.52 software (National Institutes of Health).

Cell culture and treatment. Human bronchial epithelial (Beas-2B) cells (American Type Culture Collection) were cultured in RPMI-1640 medium with 10\% FBS (Wisent, Inc.), and $1 \%$ streptomycin-penicillin antibiotics under an atmosphere of $5 \% \mathrm{CO}_{2}$ at $37^{\circ} \mathrm{C}$. The cells were pretreated with apelin-36 $(0.1,0.5$ or $1 \mu \mathrm{M})$ for $1 \mathrm{~h}$, and subsequently stimulated with LPS $(1 \mu \mathrm{g} / \mathrm{ml})$ for $6 \mathrm{~h}$. Untreated cells served as a control.

For overexpression of ASK1, recombinant full-length human ASK1 $(2 \mu \mathrm{g} / \mathrm{ml})$ was cloned into the pcDNA3.0 vector (Invitrogen; Thermo Fisher Scientific, Inc.), and then transfected into cells (at a density of 60-70\%) using Lipofectamine ${ }^{\circledR}$ 2000 (Invitrogen; Thermo Fisher Scientific, Inc.) according to the manufacturer's instructions. A pcDNA3.0 empty vector was used as a negative control (pcDNA-NC). At $48 \mathrm{~h}$ post-transfection, cells were selected for subsequent experiments.

Cell Counting Kit-8 (CCK-8) assay. To determine the cell viability, cells were seeded into 96 -well plates at a density of $4 \times 10^{4}$, and then exposed to the aforementioned treatments. 
Subsequently, cells were incubated with $50 \mu 1$ CCK-8 (Beyotime Institute of Biotechnology) working solution diluted in $0.5 \mathrm{ml}$ cell culture medium for $2 \mathrm{~h}$ under normal cell culture conditions, and then the absorbance at $450 \mathrm{~nm}$ was detected with a microplate reader.

ELISA. The concentration of apelin-36 in BALF, and the activities of inflammatory factors, including interleukin-6 (IL-6), monocyte chemoattractant protein-1 (MCP-1) and tumor necrosis factor- $\alpha$ (TNF- $\alpha$ ) in BALF and the supernatant of the cells, were detected using an ELISA assay following previously described methods (5).

Western blot analysis. In order to obtain the total protein extract, lung tissues were homogenized using $50 \mathrm{~ms}$ pulse ultrasonication at $4^{\circ} \mathrm{C}$ for 5-10 min, and total protein of the lung tissues or Beas-2B cells was extracted using RIPA lysis buffer (Thermo Fisher Scientific, Inc.). After quantification using a BCA kit (Thermo Fisher Scientific, Inc.), a total of $10 \mu \mathrm{g}$ protein samples were separated via $12 \%$ sodium dodecyl sulfate-polyacrylamide gel electrophoresis, and subsequently transferred to polyvinylidene fluoride membranes (EMD Millipore). After blocking with $5 \%$ non-fat milk at room temperature for $2 \mathrm{~h}$, the membranes were incubated with primary antibodies against Bcl-2 (cat. no. sc-7382; 1:1,000), Bax (cat. no. sc-7480; 1:1,000), caspase-3 (cat. no. sc-271759; 1:200), cleaved-caspase-3 (cat. no. sc-373730; 1:200), ASK1 (cat. no. sc-390275; 1:500), p38 (cat. no. sc-7972; 1:500), phosphorylated (p)-p38 (cat.no. sc-166182; 1:500), JNK (cat.no. sc-7345; 1:500), p-JNK (cat. no. sc-6254; 1:500), extracellular signal-regulated kinase (ERK; cat. no. sc-514302; 1:500), p-ERK (cat. no. sc-7383; 1:200) and GAPDH (cat. no. sc-47724; 1:1,000; all purchased from Santa Cruz Biotechnology, Inc.). The primary antibodies were detected using horseradish peroxidase-conjugated goat anti-rabbit (cat. no. ab6721; 1:2,000) and goat anti-mouse (cat. no. ab6789; 1:2,000; both purchased from Abcam) secondary antibodies at room temperature for $2 \mathrm{~h}$, and visualized by chemiluminescence (Thermo Fisher Scientific, Inc.). The protein levels were normalized against GAPDH. ImageJ software (v1.46r; National Institutes of Health) was used to semi-quantify the intensity of each protein band.

Reverse transcription-quantitative PCR (RT-qPCR). Total RNA from Beas-2B cells was isolated using TRIzol ${ }^{\circledR}$ reagent (Invitrogen; Thermo Fisher Scientific, Inc.) and reverse transcribed to cDNA using the PrimeScript RT Reagent Kit with gDNA Eraser (Takara Bio, Inc.), according to the manufacturer's instructions. A total of 50 ng cDNA was subsequently used for qPCR using TB Green ${ }^{\circledR}$ Fast qPCR Mix (Takara Biotechnology Co., Ltd.). The following primers were used: ASK1, forward 5'-AGACCCTGCATTTCGGGAAG-3' and reverse 5'-GCCAGATCGAAGGTCGGTAG-3'; GAPDH, forward, 5'-AGTGCCAGCCTCGTCTCATA-3' and reverse 5'-CTCGTGGTTCACACCCATCA-3'. GAPDH was used as the control. The following thermocycling conditions were used for qPCR: Initial denaturation at $95^{\circ} \mathrm{C}$ for $30 \mathrm{sec}$; and 40 cycles of $95^{\circ} \mathrm{C}$ for $5 \mathrm{sec}$ and $60^{\circ} \mathrm{C}$ for $15 \mathrm{sec}$, followed by default of melt curve (Applied Biosystems 7500; Thermo Fisher Scientific, Inc.). Differential expression of mRNA was calculated using the $2^{-\Delta \Delta \mathrm{Cq}}$ method (17).
Flow cytometric analysis. Cells were subjected to flow cytometry, based on Annexin V/PI staining (Beyotime Institute of Biotechnology). Briefly, cells at a density of $5 \times 10^{5}$ cells/well seeded in 6-well plates were collected after the aforementioned treatments, washed with PBS, and subsequently $100 \mu \mathrm{l}$ binding buffer was added. Next, $5 \mu 1$ Annexin V-FITC and $5 \mu 1$ PI were added, followed by an incubation in the dark at room temperature for $15 \mathrm{~min}$. The apoptotic rate (early and late apoptosis) was subsequently measured using a BD FACSCelesta ${ }^{\mathrm{TM}}$ flow cytometer (BD Biosciences). Data were analyzed using flow cytometry software (iSort ${ }^{\mathrm{TM}}$ Automated Cell Sorter; Thermo Fisher Scientific, Inc.).

Statistical analysis. In the present study, GraphPad Prism 6.0 (GraphPad Software, Inc.) was used for statistical analyses. All data are expressed as the mean \pm standard deviation. The differences between groups were analyzed using one-way analysis of variance followed by Tukey's multiple-comparison test. $\mathrm{P}<0.05$ was considered to indicate a statistically significant difference.

\section{Results}

Apelin-36 alleviates lung injury, inflammation and apoptosis in LPS-treated rats. First, LPS was used to induce ALI in the experimental rats, and subsequently the normal rats or LPS-induced rats were treated with or without apelin-36. Lung tissues and BALF in the different treatment groups were collected, and the concentration of apelin-36 was found to be significantly increased in the BALF of LPS-treated rats (Fig. 1A), suggesting its potential role in regulating ALI. As shown in Fig. 1B, the lung tissues of rats that underwent LPS administration showed clear pathological alterations compared with the control rats, including intra-alveolar hemorrhage, inter-alveolar septum thickening and inflammatory cell infiltration. However, in the LPS + apelin-36 group, a relatively normal lung structure and an improvement in inflammatory cell infiltration were observed. Furthermore, apelin-36 treatment alone did not cause any histological alterations of the lung tissues compared with those of the control group. Additionally, rats treated with LPS showed increased wet/dry ratios compared with the control group, which were significantly reduced upon co-treatment with apelin-36 (Fig. 1C). These data demonstrated the protective effect of apelin-36 on LPS-induced lung damage.

Subsequently, the concentrations of pro-inflammatory cytokines, including IL-6, MCP-1 and TNF- $\alpha$ in BALF, were measured. The results presented in Fig. 1D revealed that apelin-36 treatment led to a decrease in the levels of LPS-induced pro-inflammatory cytokines. As shown in Fig. 2A, lung tissues in each group were stained with TUNEL to highlight the apoptotic cells, and apelin-36 led to a significant reduction in the number of apoptotic cells compared with the LPS treatment group. The results shown in Fig. 2B further confirmed the aforementioned observations, as LPS reduced the expression of the anti-apoptotic protein $\mathrm{Bcl}-2$, whereas the expression levels of the pro-apoptotic proteins Bax and cleaved-caspase-3 were increased. The presence of apelin-36 partially recovered the expression levels of these proteins that had been altered 
A
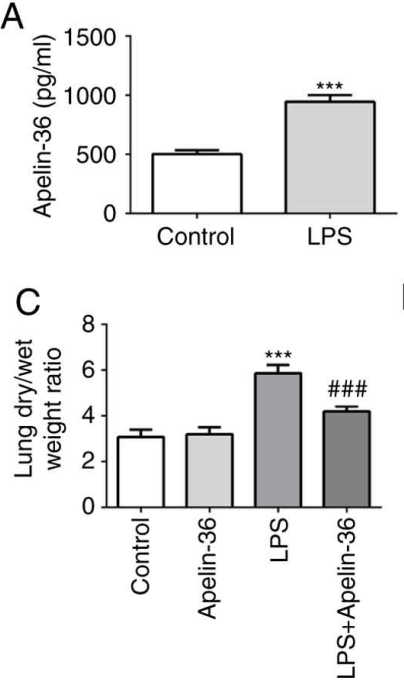

B

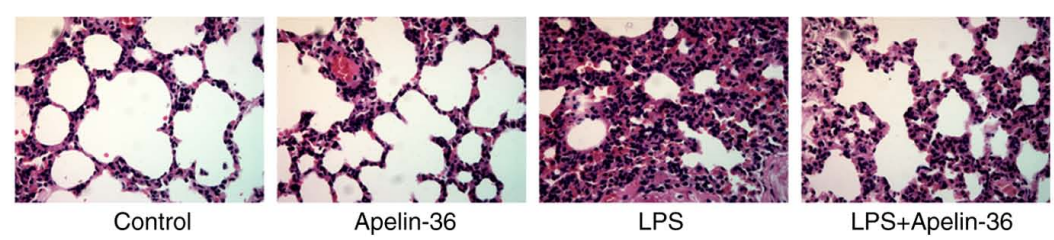

D
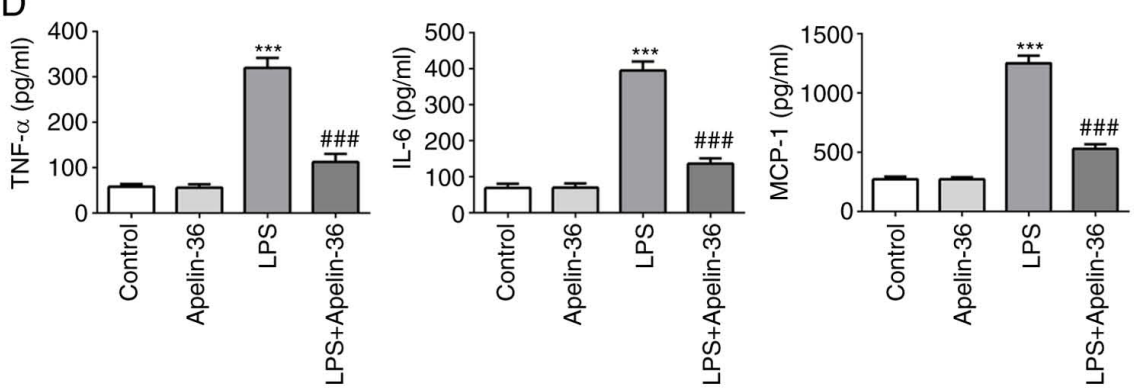

Figure 1. Apelin-36 alleviates lung injury and inflammation in LPS-treated rats. (A) The concentration of apelin-36 in the BALF of normal rats and LPS-treated rats is presented $(n=5)$. (B) Morphological changes in the lung, as determined by hematoxylin and eosin staining (magnification, $x 400)$. The sections are shown after staining with hematoxylin (blue, nuclei) and eosin (pink, muscle fibers). (C) The lung dry/wet weight ratio of rats subjected to the respective treatments ( $\mathrm{n}=5$ ). (D) The concentrations of TNF- $\alpha$, IL-6 and MCP-1 in the BALF of rats subjected to the respective treatments $(\mathrm{n}=5)$. ${ }^{* * * *} \mathrm{P}<0.001 \mathrm{vs}$. control;

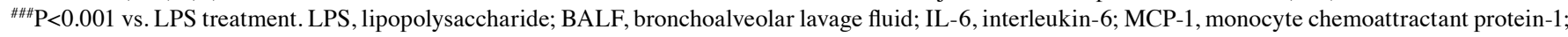
TNF- $\alpha$, tumor necrosis factor- $\alpha$.

A
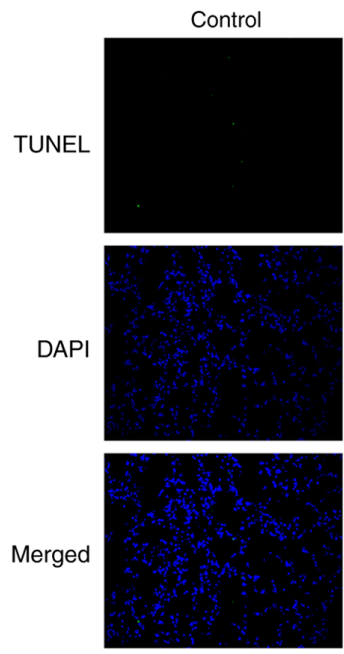

B

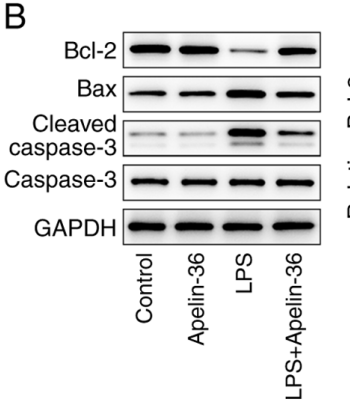

Apelin-36
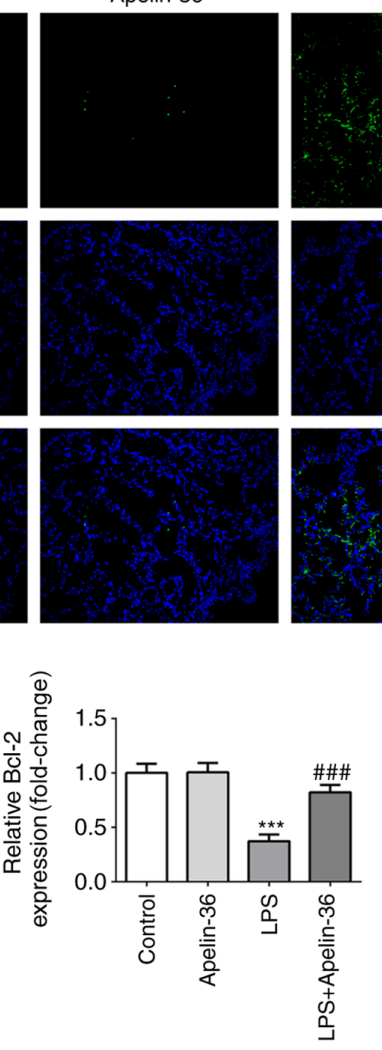

LPS
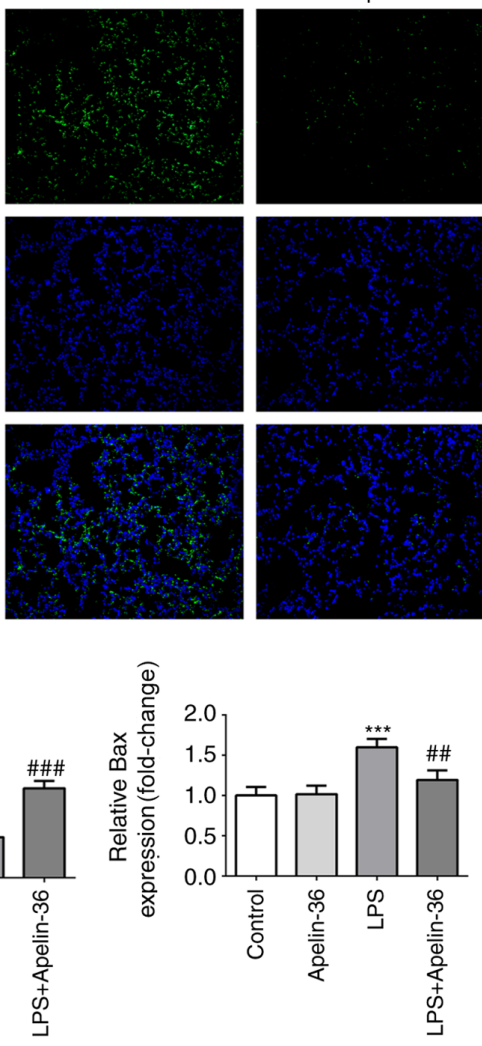

LPS+Apelin-36
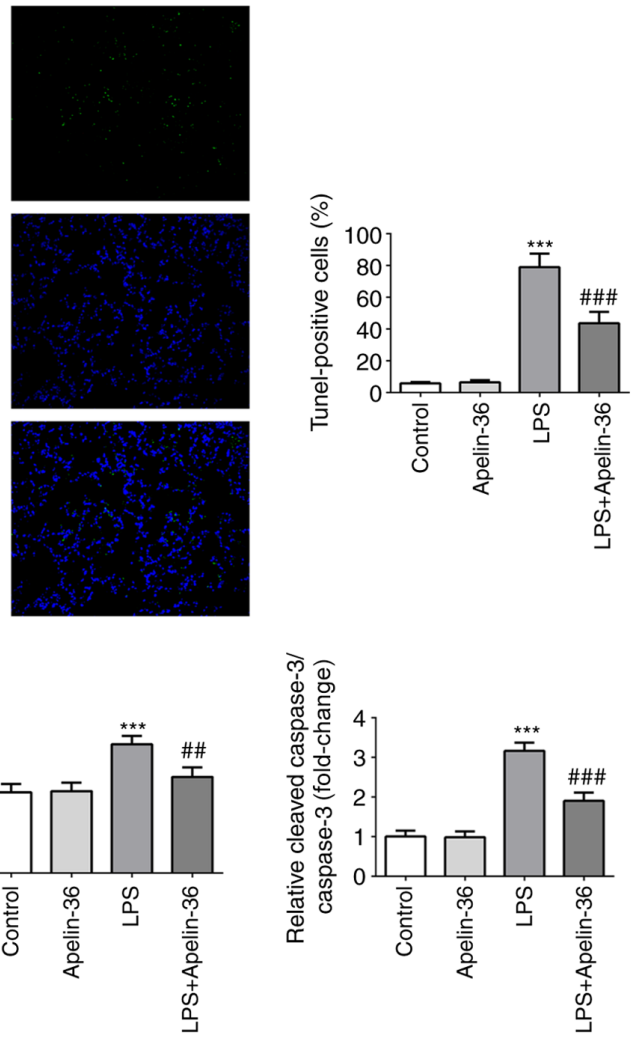

Figure 2. Apelin-36 inhibits cell apoptosis in lung tissues of LPS-treated rats. (A) Representative TUNEL staining and quantitative analysis of apoptosis in lung tissues. The dead cells are stained with green, and DAPI was used to stain the nuclei (shown in blue; magnification, x200). (B) Expression levels of the apoptosis-associated proteins, including Bcl-2, Bax and caspase-3, in the lung tissues of rats subjected to the various treatments were detected using western blotting $(\mathrm{n}=5) .{ }^{* * *} \mathrm{P}<0.001$ vs. control; ${ }^{\# \#} \mathrm{P}<0.01$ and ${ }^{\# \# \#} \mathrm{P}<0.001$ vs. LPS treatment. LPS, lipopolysaccharide.

by LPS. These findings suggested that apelin-36 was able to inhibit LPS-induced inflammation and apoptosis in the lung tissues of rats.
Apelin-36 inhibits the activation of LPS-induced ASK1/MAPK signaling. To investigate the potential underlying mechanisms of apelin-36, the expression levels of proteins associated with 

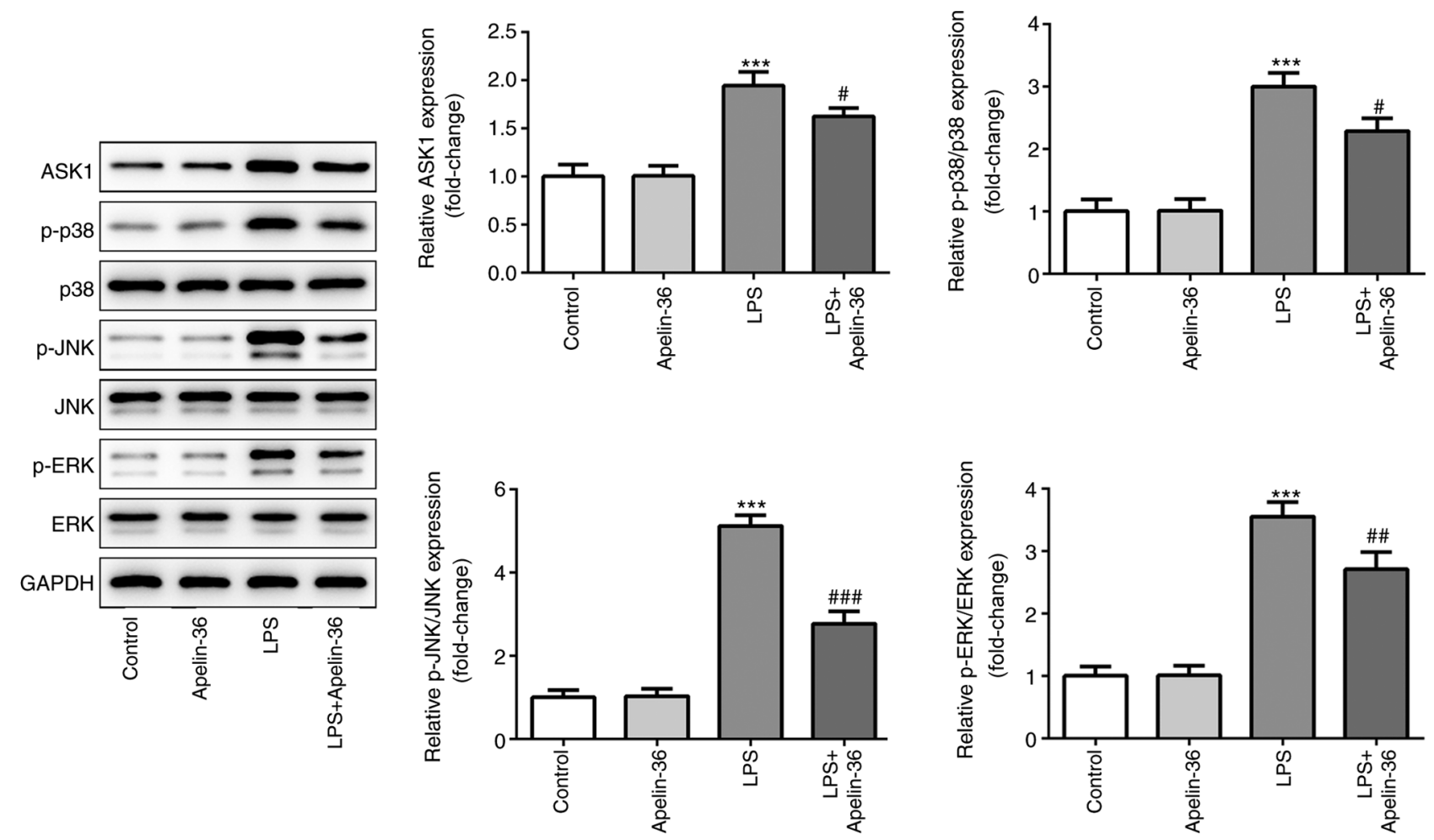

Figure 3. Apelin-36 suppresses LPS-induced activation of the ASK1/MAPK signaling pathway. Expression levels of proteins involved in ASK1/MAPK signaling, including ASK1, p-p38, p-JNK and p-ERK, in the lung tissues of rats subjected to the various treatments were detected using western blotting (n=5). ${ }^{* * * *} \mathrm{P}<0.001$ vs. control; ${ }^{\#} \mathrm{P}<0.05,{ }^{\# \#} \mathrm{P}<0.01$ and ${ }^{\# \# \#} \mathrm{P}<0.001$ vs. LPS treatment. LPS, lipopolysaccharide; $\mathrm{p}$-, phosphorylated; ASK1, apoptosis signal-regulating kinase 1; MAPK, mitogen-activated protein kinase; JNK, c-Jun N-terminal kinase; ERK, extracellular signal-regulated kinase.

the ASK1/MAPK signaling pathway were evaluated. The results presented in Fig. 3 demonstrated that LPS led to a significant increase in the expression levels of ASK1, p-p38, p-JNK and p-ERK, indicating that the ASK1/MAPK pathway was activated in the lung tissues of rats upon LPS stimulation. On the other hand, the expression levels of ASK1, p-p38, p-JNK and p-ERK were significantly reduced in the LPS + apelin-36 co-treatment groups compared with LPS treatment alone. These results suggested that apelin-36 could inhibit the LPS-induced activation of the ASK1/MAPK signaling pathway.

Overexpression of ASK1 suppresses the inhibitory effect of apelin-36 on inflammation and apoptosis. To further verify the aforementioned observations, ASK1 was overexpressed in Beas-2B cells. The results presented in Fig. 4A and B confirmed the transfection efficiency of ASK1 overexpression. As shown in Fig. 4C, apelin-36 increased the cell viability of Beas-2B cells, which had been reduced by LPS, in a concentration-dependent manner. As $1 \mu \mathrm{M}$ apelin-36 had the most significant effects on cell viability, it was chosen as the concentration for subsequent experiments. Then, cells overexpressed with or without ASK1 were exposed to LPS, $1 \mu \mathrm{M}$ apelin-36 or LPS + apelin-36 $(1 \mu \mathrm{M})$. LPS treatment led to a significant increase in the levels of TNF- $\alpha$, IL- 6 and MCP-1, whereas in the apelin-36 co-treatment group, the levels of these inflammatory cytokines were significantly reduced (Fig. 4D). However, by contrast, the overexpression of ASK1 led to an increase in the levels of these cytokines compared with the LPS + apelin-36 group, suggesting that ASK1 overexpression could suppress the inhibitory effect of apelin-36 on LPS-induced inflammation in lung cells.

Similarly, as presented in Fig. 5A, LPS treatment resulted in an increase in cell apoptosis, which was reversed by apelin-36 co-treatment, although this was increased by the overexpression of ASK1. Furthermore, the expression levels of apoptosis-associated proteins in the different groups were observed. It was found that LPS led to a decrease in Bcl-2 expression, whereas the expression levels of Bax and cleaved-caspase-3 were increased (Fig. 5B), indicating the occurrence of apoptosis induced by LPS. Apelin-36 co-treatment led to a partial recovery in the balance of these proteins, although overexpression of ASK1 reversed the effects of apelin-36 on these apoptosis-associated proteins. Taken together, the aforementioned results suggested that overexpression of ASK1 was able to suppress the inhibitory effects of apelin-36 on LPS-induced inflammation and apoptosis in Beas-2B cells.

\section{Discussion}

The involvement of apelin proteins in various diseases, including cardiovascular diseases, diabetes, obesity and neurological disorders $(11,12,18)$, has been extensively reported. To the best of our knowledge, the present study was the first to investigate the modulatory effects of apelin-36 on LPS-induced ALI, and demonstrate that apelin-36 could protect against LPS-induced lung injury both in vivo and in vitro. Additionally, the associated mechanism may involve the inhibition of ASK1/MAPK signaling. 
A
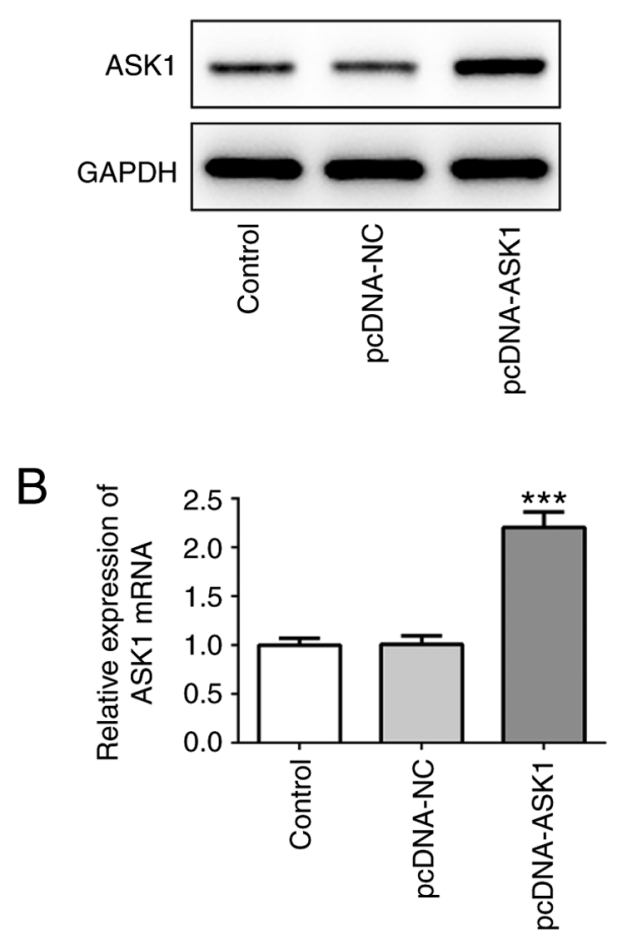
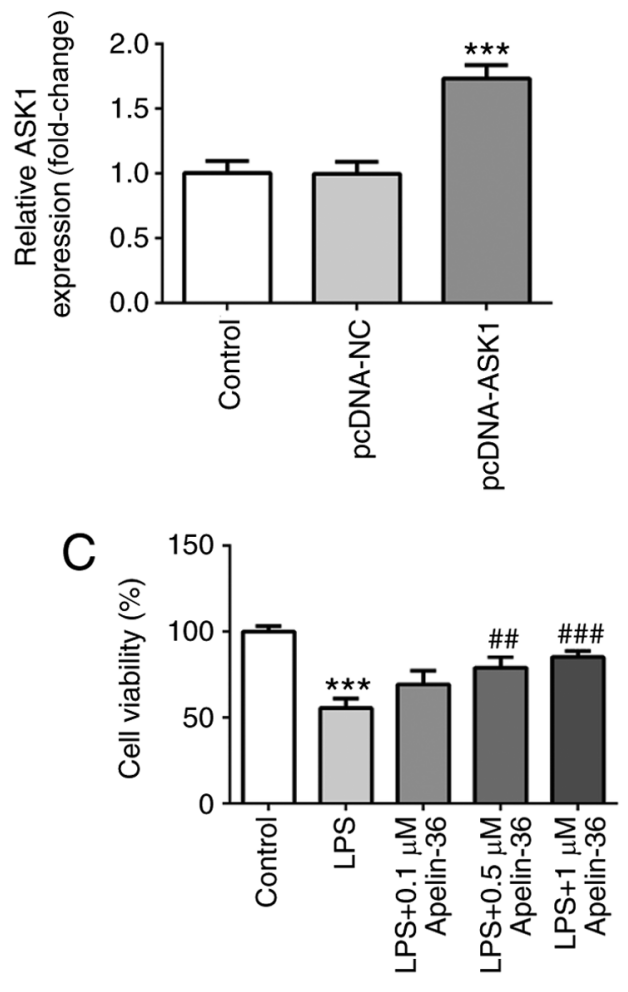
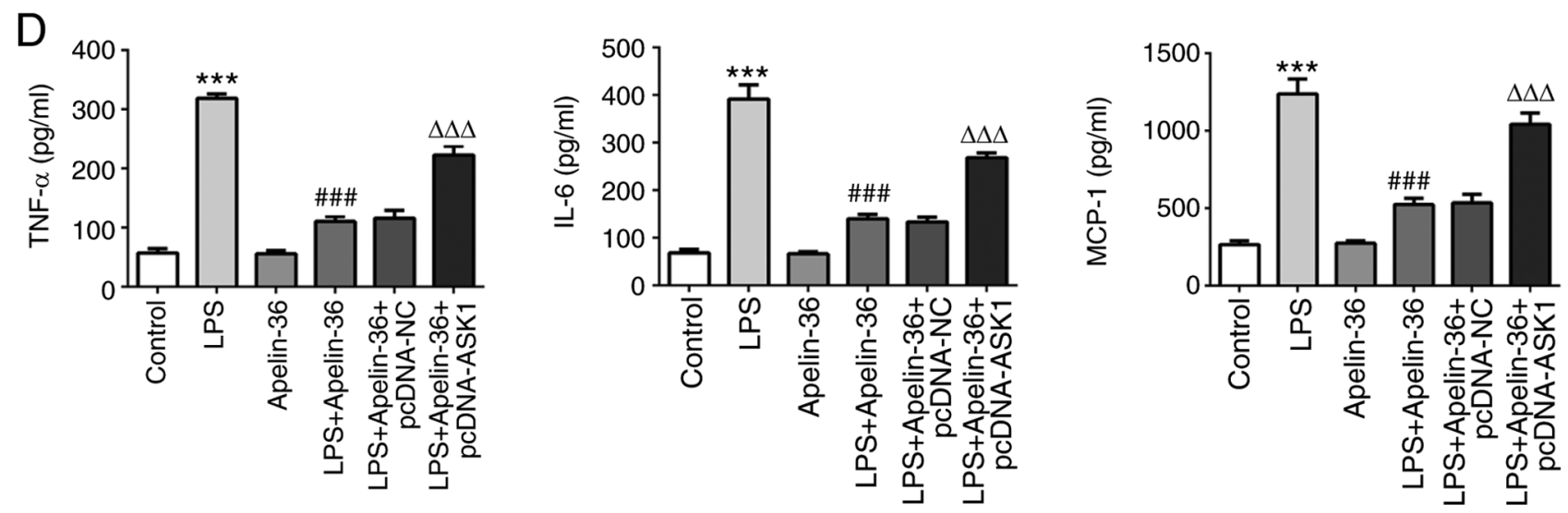

Figure 4. Overexpression of ASK1 blocks the inhibitory effect of apelin-36 on inflammation. The (A) protein and (B) mRNA expression of ASK1 in Beas-2B cells transfected with pcDNA-NC or pcDNA-ASK1, data were obtained relative to control group ( $\mathrm{n}=3$ ). (C) The cell viability of Beas-2B cells treated with or without different concentrations of apelin-36, in the presence of LPS stimulation (n=3). (D) The concentrations of TNF- $\alpha$, IL-6 and MCP-1 in the culture medium of Beas-2B cells that were subjected to the aforementioned treatments $(\mathrm{n}=3) .{ }^{* * *} \mathrm{P}<0.001$ vs. control; ${ }^{\# \#} \mathrm{P}<0.01$ and ${ }^{\# \# \#} \mathrm{P}<0.001$ vs. LPS treatment; ${ }^{\Delta \Delta \Delta} \mathrm{P}<0.001$ vs. LPS + apelin-36 + pcDNA-NC. ASK1, apoptosis signal-regulating kinase 1; NC, negative control; LPS, lipopolysaccharide; IL-6, interleukin-6; MCP-1, monocyte chemoattractant protein-1; TNF- $\alpha$, tumor necrosis factor- $\alpha$.

There is an increasing body of evidence that supports the role of inflammation and apoptosis in the initiation and progression of ALI. ALI is a prevailing inflammatory lung disease characterized by the increased production of pro-inflammatory mediators, infiltration of inflammatory cells, and apoptosis of alveolar epithelial cells $(19,20)$. The role of endotoxins, especially LPS, has been well recognized in the pathogenesis of ALI. LPS is a potent activator of Toll-like receptor 4 , triggering the $\mathrm{NF}-\kappa \mathrm{B}$ signaling pathway, thereby producing pro-inflammatory molecules, such as IL-6, IL-1 $\beta$ and TNF- $\alpha$ (15). Consistent with this previous study, the results of the present study showed that LPS could clearly cause pathological manifestations, including intra-alveolar hemorrhage, inter-alveolar septum thickening, inflammatory cell infiltration, pulmonary edema and cell apoptosis, in both the lung tissues of rats and lung bronchial epithelial cells. Therefore, controlling aberrant inflammation and apoptosis is considered to be an effective strategy to attenuate lung injury.

The APJ receptor and its endogenous ligand apelin protein may be detected in endothelial cells, cardiomyocytes and vascular smooth muscle cells, and their interaction helps to maintain the normal function of the body; dysregulation of this process is associated with the pathophysiological processes of various diseases $(7,12,21)$. For example, apelin-36 can be produced and released by the vascular endothelium, subsequently binding to neighboring APJ receptors. It can activate endothelial nitric oxide synthase to stimulate nitric oxide production, the release of which helps to maintain 
A
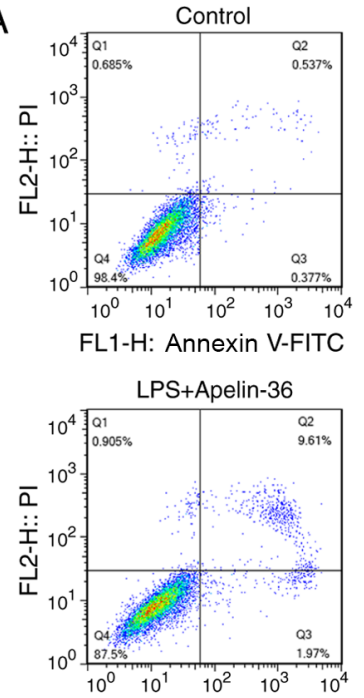

FL1-H: Annexin V-FITC

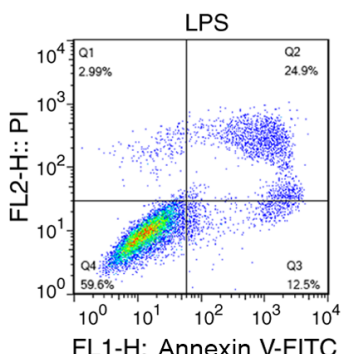

FL1-H: Annexin V-FITC

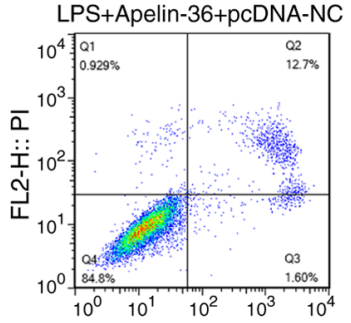

FL1-H: Annexin V-FITC

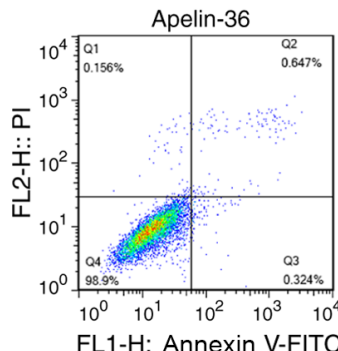

FL1-H: Annexin V-FITC

LPS+Apelin-36+pcDNA-ASK1

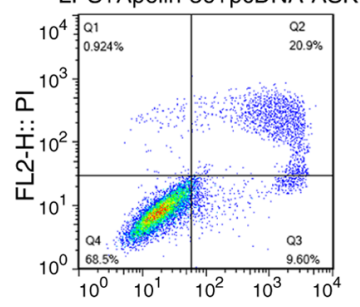

FL1-H: Annexin V-FITC
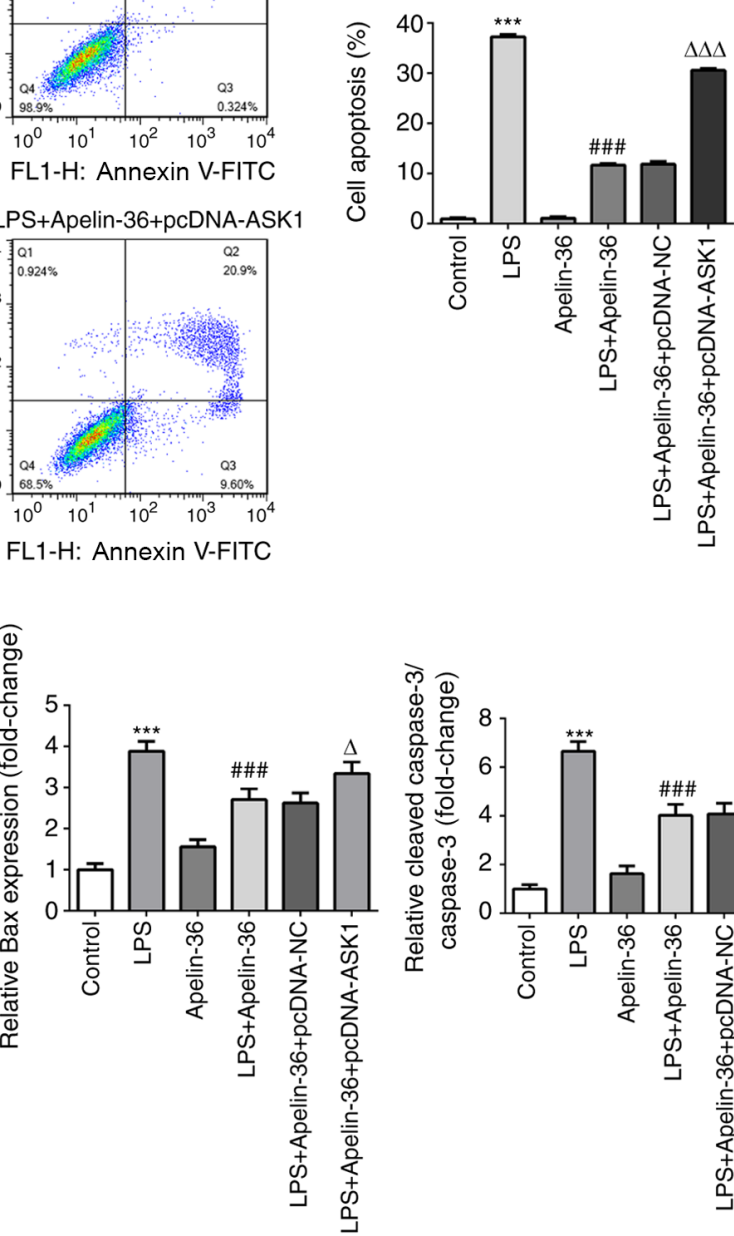

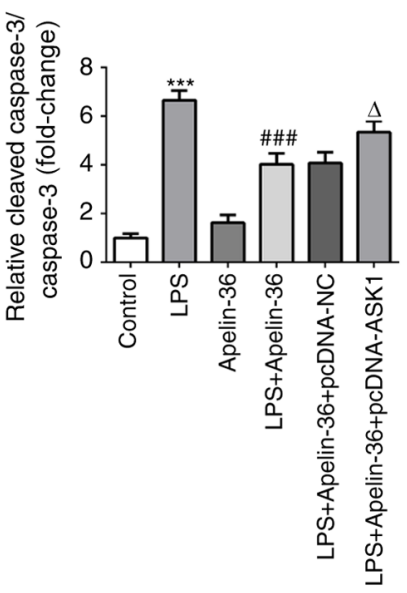

Figure 5. Overexpression of ASK1 suppresses the inhibitory effect of apelin-36 on cell apoptosis. (A) Representative flow cytometry and quantitative analysis of apoptosis in the different groups $(\mathrm{n}=3)$. (B) The expression of apoptosis-related proteins, including Bcl-2, Bax and caspase-3, in Beas-2B cells in the various treatment groups $(\mathrm{n}=3)$. ${ }^{* * * *} \mathrm{P}<0.001$ vs. control; ${ }^{\# \#} \mathrm{P}<0.001$ vs. LPS treatment; ${ }^{\Delta} \mathrm{P}<0.05$ and ${ }^{\Delta \Delta \Delta} \mathrm{P}<0.001$ vs. LPS + apelin-36 + pcDNA-NC. ASK1, apoptosis signal-regulating kinase 1 ; NC, negative control; LPS, lipopolysaccharide.

vascular tone and regulate blood pressure stability (22). The role of apelin-36 in the cardiovascular system has become a research hotspot. For instance, apelin-36 can improve myocardial ischemia, and its mechanism of action may be associated with the reduction of mitochondrial damage of myocardial cells under hypoxic conditions and protection of the energy supply of myocardial cells $(18,23)$. In brain injury, apelin-36 can significantly reduce the expression levels of apoptosis markers, including caspase- 3 and Bax, and it may also inhibit activation of the phosphoinositide 3-kinase signaling pathway, reduce cell apoptosis, and reduce cerebral cortex damage in hypoxic-ischemic neonatal rats (24). In the present study, it was found that apelin-36 was upregulated in the BALF of rats subjected to LPS treatment. This finding was in accordance with a previous study, in which elevated levels of apelin-36 in the plasma, BALF and lung tissue were confirmed in rats with ARDS, indicating the important pathophysiological function of apelin-36 in lung injury (5). To further confirm the role of apelin-36, it was administered to normal rats or LPS-induced rats. Apelin-36 administration was shown to have no obvious influence on the structure and function of the lungs of normal rats, although it could markedly alleviate the pathological alterations in lung tissues, lung injury, pulmonary edema, generation of inflammatory cytokines and cell apoptosis induced by LPS both in rats and in Beas-2B cells. These results suggested that apelin-36 could protect against LPS-induced lung injury without producing any notable side effects.

ASK1 is a member of the MAPK family. MAPKs induce cell apoptosis and activate downstream signaling pathways of MAPKs, JNKs and p38 MAPKs. ASK1 is involved in numerous types of stress response, including apoptosis, and ASK1 has been reported to be associated with numerous diseases, including lung injury $(25,26)$. In the present study, it was shown that apelin-36 could downregulate the LPS-induced expression levels of ASK1, p-p38, p-JNK and p-ERK, these proteins all being members of the MAPK family that can be activated in response to LPS. These results indicated that the actions of apelin-36 may be dependent on the inhibition of ASK1/MAPK signaling. To verify this hypothesis, ASK1 was overexpressed in cells that were subsequently co-treated with LPS and 
apelin-36. The results demonstrated that the overexpression of ASK1 could suppress the inhibitory effects of apelin-36 on LPS-induced inflammation and apoptosis to a significant extent, confirming that apelin-36 exerted anti-inflammatory and anti-apoptotic effects in LPS-induced lung injury via inhibiting ASK1. However, the overexpression of ASK1 did not completely eliminate the effects of apelin-36, suggesting that other targets are involved in the actions of apelin-36. The MAPK family consists of a large number of members, including p-38, JNK and ERK, and whether apelin-36 is able to also directly target these proteins, or whether the targeting of ASK1 thereby affects p-38, JNK and ERK downstream in the signaling pathway, remains to be elucidated.

Taken together, the present study demonstrated the potential pharmacological effects of apelin-36 in ALI, and the possible underlying mechanism. The results highlighted that apelin-36 may be a potential novel candidate for the treatment of injurious inflammatory responses and apoptosis in ALI via targeting ASK1, thereby inhibiting ASK1/MAPK signaling. However, further studies are needed to investigate the specific relationship between apelin-36 and ASK1.

\section{Acknowledgements}

Not applicable.

\section{Funding}

This study was funded by the National Natural Science Foundation of China (grant no. 81860056), the Key Scientific Research Project of Health Department of Ningxia Hui Autonomous Region (grant no. 2019-NW-018), and The Scientific Research Project of Ningxia Hui Autonomous Region People's Hospital (grant no. 201918).

\section{Availability of data and materials}

All data generated or analyzed during this study are included in this published article.

\section{Authors' contributions}

QH, YW and DL conceived and designed the study. QH, YW, HY, JW and JZ acquired and analyzed the data. DL prepared the draft of the manuscript, including the figures. All authors read and approved the final manuscript.

\section{Ethics approval and consent to participate}

All experimental procedures were approved by the Ethical Committee on Animal Research at the People's Hospital of Ningxia Hui Autonomous Region (Yinchuan, China).

\section{Patient consent for publication}

Not applicable.

\section{Competing interests}

The authors declare that they have no competing interests.

\section{References}

1. Butt Y, Kurdowska A and Allen TC: Acute lung injury: A clinical and molecular review. Arch Pathol Lab Med 140: 345-350, 2016.

2. Thompson BT, Chambers RC and Liu KD: Acute respiratory distress syndrome. N Engl J Med 377: 562-572, 2017.

3. Hughes KT and Beasley MB: Pulmonary manifestations of acute lung injury: More than just diffuse alveolar damage. Arch Pathol Lab Med 141: 916-922, 2017.

4. Monsel A,Zhu YG, Gudapati V,Lim H and Lee JW: Mesenchymal stem cell derived secretome and extracellular vesicles for acute lung injury and other inflammatory lung diseases. Expert Opin Biol Ther 16: 859-871, 2016.

5. Fan XF, Xue F, Zhang YQ, Xing XP, Liu H, Mao SZ, Kong XX, Gao YQ, Liu SF and Gong YS: The Apelin-APJ axis is an endogenous counterinjury mechanism in experimental acute lung injury. Chest 147: 969-978, 2015.

6. Vinel C, Lukjanenko L, Batut A, Deleruyelle S, Pradère JP, Le Gonidec S, Dortignac A, Geoffre N, Pereira O, Karaz S, et al: The exerkine apelin reverses age-associated sarcopenia. Nat Med 24: 1360-1371, 2018

7. Cheng J, Luo X, Huang Z and Chen L: Apelin/APJ system: A potential therapeutic target for endothelial dysfunction-related diseases. J Cell Physiol 234: 12149-12160, 2019.

8. Zhu J, Dou S, Jiang Y, Bai B, Chen J, Wang C and Cheng B: Apelin-36 exerts the cytoprotective effect against $\mathrm{MPP}^{+}$-induced cytotoxicity in SH-SY5Y cells through PI3K/Akt/mTOR autophagy pathway. Life Sci 224: 95-108, 2019.

9. Zhu J, Dou S, Wang C, Jiang Y, Wang C and Cheng B: Apelin-36 mitigates MPTP/MPP ${ }^{+}$-induced neurotoxicity: Involvement of $\alpha$-synuclein and endoplasmic reticulum stress. Brain Res 1721: 146334, 2019.

10. Folino A, Montarolo PG, Samaja M and Rastaldo R: Effects of apelin on the cardiovascular system. Heart Fail Rev 20: 505-518, 2015.

11. Castan-Laurell I, Dray C, Attané C, Duparc T, Knauf C and Valet P: Apelin, diabetes, and obesity. Endocrine 40: 1-9, 2011.

12. Antushevich $\mathrm{H}$ and Wójcik M: Review: Apelin in disease. Clin Chim Acta 483: 241-248, 2018.

13. Zhu J, Gao W, Shan X, Wang C, Wang H, Shao Z, Dou S, Jiang Y, Wang $C$ and Cheng B: Apelin-36 mediates neuroprotective effects by regulating oxidative stress, autophagy and apoptosis in MPTP-induced Parkinson's disease model mice. Brain Res 1726: $146493,2020$.

14. Ko IG, Hwang JJ, Chang BS, Kim SH, Jin JJ, Hwang L, Kim CJ and Choi CW: Polydeoxyribonucleotide ameliorates lipopolysaccharide-induced acute lung injury via modulation of the MAPK/NF-kappaB signaling pathway in rats. Int Immunopharmacol 83: 106444, 2020.

15. Jin Y, Qian J, Ju X, Bao X, Li L, Zheng S, Chen X, Xiao Z, Chen $\mathrm{X}, \mathrm{Zhu} \mathrm{W}$, et al: Osthole protects against acute lung injury by suppressing NF- $\mathrm{BB}-$ Dependent Inflammation. Mediators Inflamm 2018: 4934592, 2018.

16. Zhou J, Hu R, Jing S, Xue X and Tang W: Activated protein C inhibits lung injury induced by LPS via downregulating MAPK signaling. Exp Ther Med 16: 931-936, 2018.

17. Livak KJ and Schmittgen TD: Analysis of relative gene expression data using real-time quantitative PCR and the 2(-Delta Delta C(T)) method. Methods 25: 402-408, 2001.

18. Yu XH, Tang ZB, Liu LJ, Qian H, Tang SL, Zhang DW, Tian GP and Tang CK: Apelin and its receptor APJ in cardiovascular diseases. Clin Chim Acta 428: 1-8, 2014.

19. Liu ZF, Zheng D, Fan GC, Peng T and Su L: Heat stress prevents lipopolysaccharide-induced apoptosis in pulmonary microvascular endothelial cells by blocking calpain/p38 MAPK signalling. Apoptosis 21: 896-904, 2016.

20. Cox R Jr, Phillips O, Fukumoto J, Fukumoto I, Parthasarathy PT, Arias S, Cho Y, Lockey RF and Kolliputi N: Enhanced resolution of hyperoxic acute lung injury as a result of aspirin triggered resolvin D1 treatment. Am J Respir Cell Mol Biol 53: 422-435, 2015.

21. Hu H, He L, Li L and Chen L: Apelin/APJ system as a therapeutic target in diabetes and its complications. Mol Genet Metab 119: 20-27, 2016.

22. Mughal A and O'Rourke ST: Vascular effects of apelin: Mechanisms and therapeutic potential. Pharmacol Ther 190: 139-147, 2018. 
23. Pisarenko O, Shulzhenko V, Studneva I, Pelogeykina Y, Timoshin A, Anesia R, Valet P, Parini A and Kunduzova O: Structural apelin analogues: Mitochondrial ROS inhibition and cardiometabolic protection in myocardial ischaemia reperfusion injury. Br J Pharmacol 172: 2933-2945, 2015.

24. Gu Q, Zhai L, Feng X, Chen J, Miao Z, Ren L, Qian X, Yu J, Li Y, $\mathrm{Xu} \mathrm{X}$ and Liu CF: Apelin-36, a potent peptide, protects against ischemic brain injury by activating the PI3K/Akt pathway. Neurochem Int 63: 535-540, 2013.

25. Noguchi T, Ishii K, Fukutomi H, Naguro I, Matsuzawa A, Takeda $\mathrm{K}$ and Ichijo $\mathrm{H}$ : Requirement of reactive oxygen species-dependent activation of ASK1-p38 MAPK pathway for extracellular ATP-induced apoptosis in macrophage. J Biol Chem 283: 7657-7665, 2008.
26. Han J, Lv W, Sheng H, Wang Y, Cao L, Huang S, Zhu L and Hu J: Ecliptasaponin A induces apoptosis through the activation of ASK1/JNK pathway and autophagy in human lung cancer cells. Ann Transl Med 7: 539, 2019.

(i) (9) This work is licensed under a Creative Commons EY No ND Attribution-NonCommercial-NoDerivatives 4.0 International (CC BY-NC-ND 4.0) License. 